\title{
Coupling Relationship of Urban Development and the Eco-Environment in Guanzhong Region, China
}

\author{
Jian Wang ${ }^{1,2}$, Mudan Zhao ${ }^{1,2, *}$, Wei Zhong ${ }^{1,2}$, Jianbo Li $^{1,2}$ and Chunyan Zheng ${ }^{3}$ \\ 1 College of Urban and Environmental Sciences, Northwest University, Xi'an 710127, China; \\ wang_jian@stumail.nwu.edu.cn (J.W.); zhongwei@stumail.nwu.edu.cn (W.Z.); \\ 201931936@stumail.nwu.edu.cn (J.L.) \\ 2 Shaanxi Remote Sensing and GIS Engineering Research Center, Xi'an 710127, China \\ 3 College of Urban and Environmental Sciences, Hubei Normal University, Huangshi 435002, China; \\ a638326@stu.hbnu.edu.cn \\ * Correspondence: zmudan@nwu.edu.cn; Tel.: +86-131-1040-1900
}

Citation: Wang, J.; Zhao, M.; Zhong, W.; Li, J.; Zheng, C. Coupling Relationship of Urban Development and the Eco-Environment in Guanzhong Region, China. Sustainability 2022, 14, 2969. https://doi.org/10.3390/su14052969 Academic Editors: Hongbin Deng and Shuxin Mao

Received: 18 January 2022

Accepted: 2 March 2022

Published: 3 March 2022

Publisher's Note: MDPI stays neutral with regard to jurisdictional claims in published maps and institutional affiliations.

Copyright: (C) 2022 by the authors. Licensee MDPI, Basel, Switzerland. This article is an open access article distributed under the terms and conditions of the Creative Commons Attribution (CC BY) license (https:// creativecommons.org/licenses/by/ $4.0 /)$.

\begin{abstract}
Since the 21st century, China's rapid urban development has had profound impacts on the regional eco-environment and posed severe threats to regional sustainable development. In order to scientifically evaluate the coupling relationship between urban development and the eco-environment in the Guanzhong region, in this paper, by combining nighttime lighting data and MODIS data in 2001, 2010 and 2018, we applied an overall coupling model and a coordination model to discuss the spatial-temporal coupling and coordination relationship between urban development and the ecoenvironment. The results showed the following: (1) From 2001 to 2018, the urbanization development in the Guanzhong region significantly improved and the links between cities were continuously strengthened, but the degree of contact still needed to be improved. (2) The eco-environment quality in the Guanzhong region slightly increased, but the overall level was low. The structure of the ecoenvironment quality grade changed greatly, and "Good" grades changed to "Very Good". (3) During 2001-2018, the overall coupling situation between urban development and the eco-environment strengthened and the degree of coordination increased. The coupling coordinator subtype gradually transformed from system balanced development into system balanced development, the ecology lag type, and the urban development lag type coexisting phenomenon. The results of the study suggest that future urban development planning and ecological protection policies need to take the coordinated coupling between urban development and the eco-environment into account.
\end{abstract}

Keywords: urban development; eco-environment; coordinated coupling; principal component analysis; Guanzhong region

\section{Introduction}

With the rapid development of China's social economy, the process of urbanization is also accelerating. It is expected that the total urban population will reach $70 \%$ by 2050 [1]. The expansion of the urban scale changes the physical properties of the urban underlying surface, and an artificial impervious surface replaces the original natural surface [2]. This is an important reason for the increasingly serious urban ecological environment problems, such as cultivated land reduction, air pollution, water pollution, and urban heat islands [3]. In recent years, the eco-environment problems caused by urban development have become the focus of the international community [4]. Therefore, it is of great significance to explore the interaction and spatial relationship between urban development and the eco-environment to improve the urban ecological environment, optimize the quality of urbanization, and realize regional sustainable development.

Research on the relationship between regional urbanization and the eco-environment is one of the hotspots in the field of earth science and sustainable development $[5,6]$. This 
research has had produced key findings on theoretical methods, such as the environmental Kuznets curve (EKC) [7,8], the tele-coupling theory [9], the footprint model [10], the coupling coordination model (CCDM) [11,12], etc. Among them, the EKC and CCDM are widely used. However, the EKC has some disadvantages, such as limited applicability and it fails to address the interaction between urban development and the ecoenvironment [13-15]. Some scholars prefer to use the CCDM to analyze the interaction between them [16,17]. At present, relevant scholars have carried out a large number of studies on the coupling relationship between urban development and the eco-environment in China. For example, Liu et al. studied the coupling relationship between urbanization and the eco-environment in 30 provinces of China in the past ten years and found that the degree of coupling and coordination between them was increasing, which is basically consistent with previous research $[14,18]$. Cai et al. analyzed the coupling degree of new urbanization and the agricultural eco-environment in 31 provinces of China and found that the coordination degree was still in the confrontation stage [19]. Feng et al. explored the coupling degree of urbanization and ecological factor energy efficiency in Hebei Province, China, and found that the coupling degree of urbanization and ecological factor energy efficiency was transitioning from the middle-low coupling stage to the middle-high coupling stage [20]. However, based on the above research, it is evident that there are still some limitations in the existing research.

Firstly, most studies mainly use statistical data to reflect the urbanization and ecoenvironment of a region, from which it is difficult to reflect the internal spatial information of an administrative region [11,20]. Moreover, statistical data are difficult to obtain and they are strongly subjective, which may lead to differences between the results and reality [21]. Secondly, the existing research evaluation system has limitations [22] and cannot provide a theoretical reference for high-quality developments outside the study area [5]. In addition, the existing research focuses on the type of coordinated coupling between urbanization and the eco-environment, ignoring the overall coordinated trend change of urban development and the eco-environment.

With the rapid development of remote sensing technology, satellite observations have the advantages of a wide area, strong time series, and repeatable observation, which greatly promote the monitoring of regional eco-environments and urbanization evaluations [23]. In 2018, $\mathrm{Xu}$ used the principal component analysis method to construct the remote sensing ecological index (RSEI) with the help of four remote sensing ecological elements: greenness, heat, wetness, and dryness [24]. This index has the advantages of high efficiency, objectivity, and accuracy in monitoring the changes in regional eco-environment quality, and it has been widely used in the evaluation of urban eco-environments [25-27]. In addition, the nighttime light (NTL) observed by the spaceborne sensor can objectively and accurately reflect regional human activities from the grid scale [28,29]. Many scholars have confirmed that the pixel value of the NLI has a significant correlation with human activity indicators, such as economic development [30], population density [31], energy consumption [32], and urbanization level [33]. Therefore, this paper establishes an evaluation system based on multi-source remote sensing data to fully understand the coupling relationship between urbanization and the eco-environment.

The Guanzhong region is the core area of the Guanzhong-Tianshui Economic Zone established in China's Western Development Plan (CWDP), and it is an important bearing region for planning the Silk Road Economic Belt. Since the implementation of CWDP, the scale of urbanization in the region has expanded rapidly and man-land contradiction has become increasingly prominent. How to balance eco-environment protection and realize regional sustainable development in the process of urbanization has become an important problem in the Guanzhong region. Therefore, based on day and night remote sensing images, this paper discusses the coupling relationship between urban development and the eco-environment in the Guanzhong region (Figure 1). The specific purposes of this study were: (1) to monitor the spatial-temporal differentiation of urban development and the eco-environment in the Guanzhong region from 2001 to 2018, and (2) to assess the coupling 
coordination of urban development and the eco-environment in the Guanzhong region from 2001 to 2018.

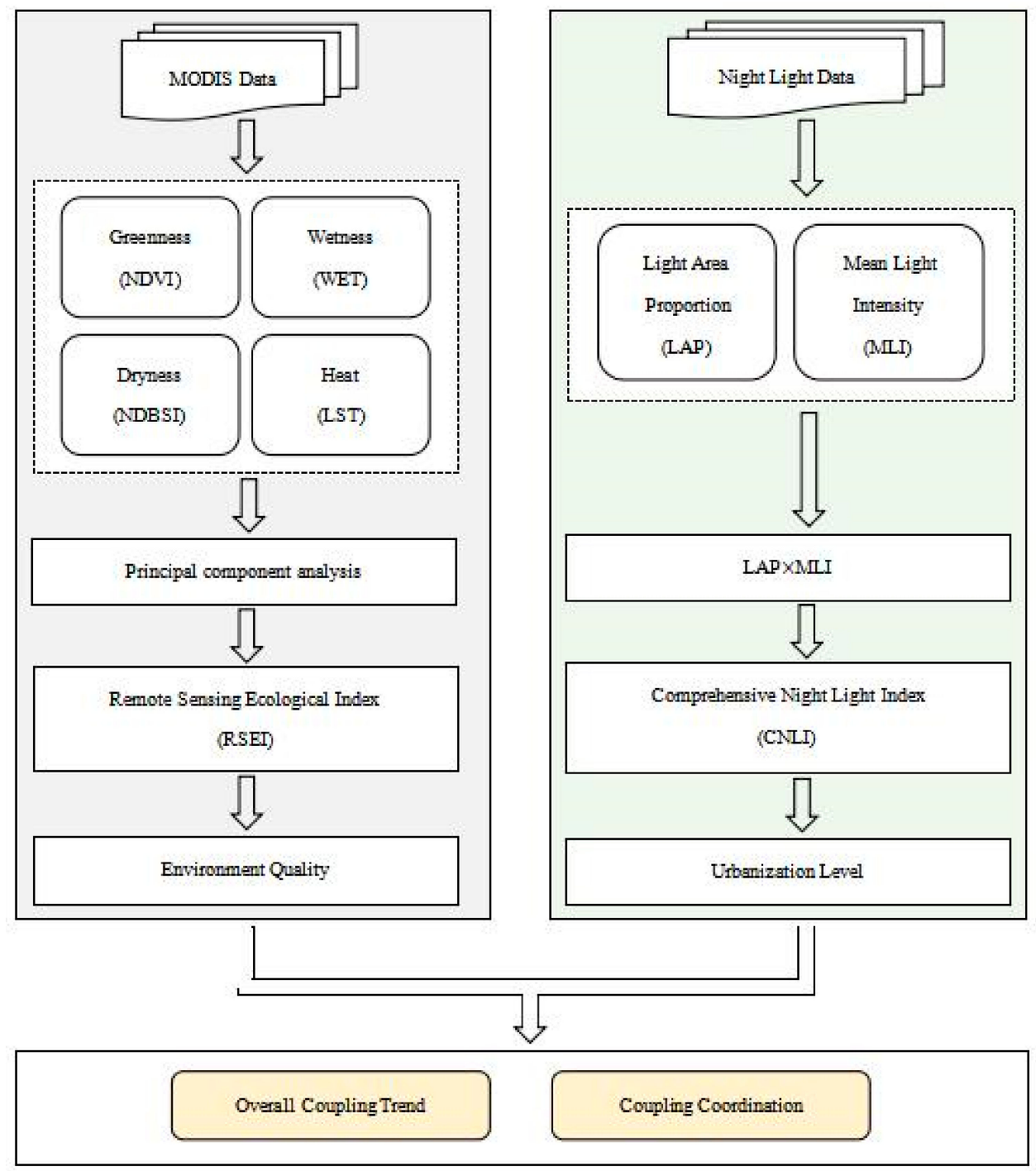

Figure 1. Overview of the research framework.

\section{Materials and Methods}

\subsection{Study Area}

The Guanzhong region $\left(106^{\circ} 30^{\prime}-110^{\circ} 60^{\prime} \mathrm{E}, 33^{\circ} 30^{\prime}-36^{\circ} 10^{\prime} \mathrm{N}\right)$ is located in the middle of Shaanxi Province, China, north of the Loess Plateau and south of the Qinling Mountains (Figure 2). The total area is $55,194.35 \mathrm{~km}^{2}$. The Guanzhong region belongs to a temperate monsoon climate, with a cold and dry winter in addition to a hot and rainy summer [34]. By 2017 the total population of the region was 24.1964 million and the GDP was CNY 1.69 trillion, accounting for $63.10 \%$ and $64.61 \%$, respectively, of Shaanxi Province. The Guanzhong region mainly includes five prefecture-level cities, Xi'an, Tongchuan, Weinan, Xianyang, and Baoji, and a total of 54 counties. The urban developments in the Guanzhong region have important strategic significance for the urban development planning of the whole province and even the whole country. Therefore, analyzing the relationship between urbanization and the eco-environment in the Guanzhong region is an important way to explore regional high-quality development. 


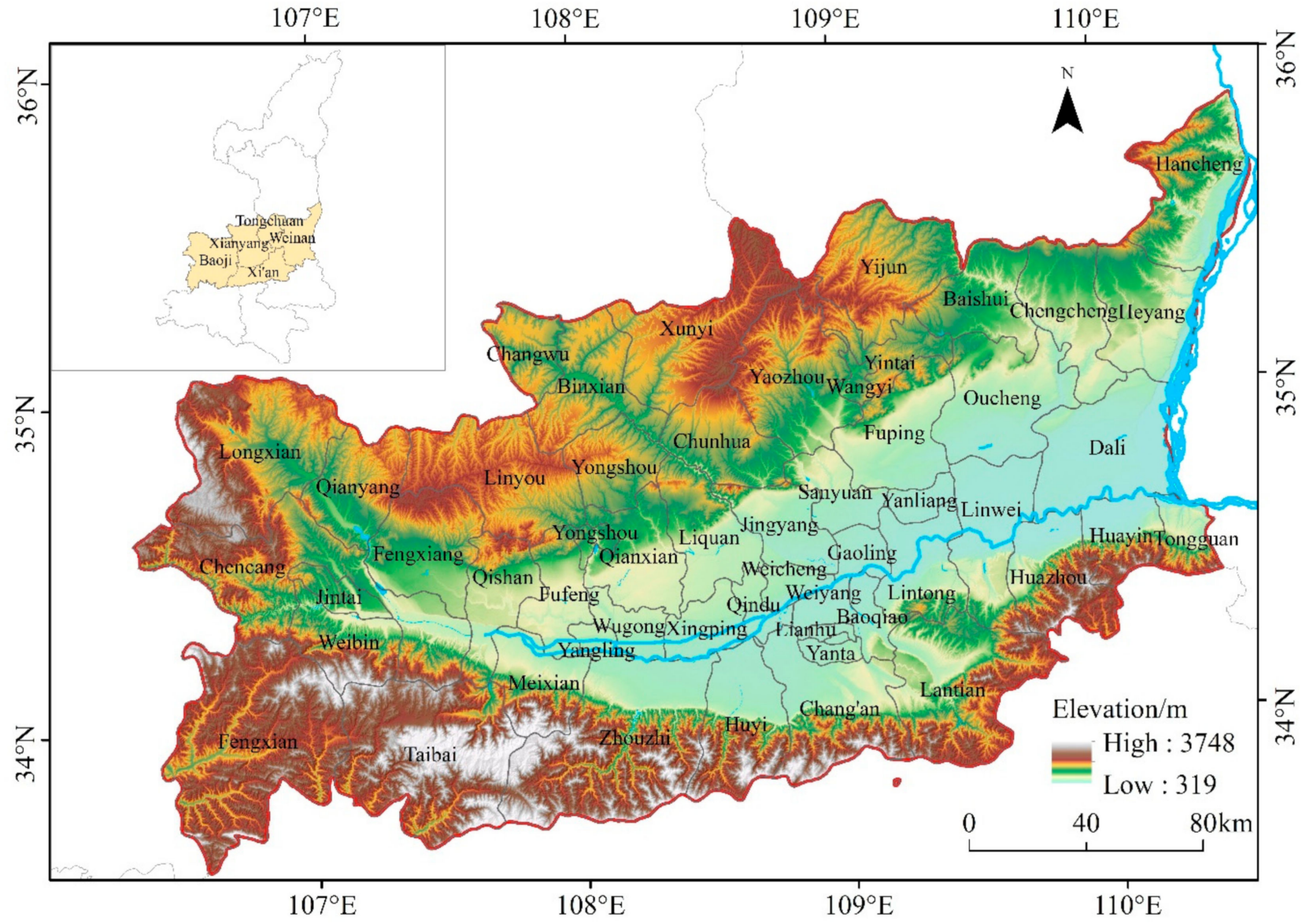

Figure 2. Location of the Guanzhong region, China.

\subsection{Data Source}

\subsubsection{Night Light Data}

At present, the main sources of nighttime light data are DMSP/OLS, NPP/VIIRS, and Luojia 1. Among them, DMSP/OLS and NPP/VIIRS are the most widely used data sources; therefore, this paper selects global stable night light data from DMSP/OLS in 2000 and 2010 and NPP-VIIRS in 2018, both of which come from the National Geophysical Data Center (https: / /ngdc.noaa.gov/eog/dmsp/downloadV4composites.html, accessed on 2 February 2022).

\subsubsection{MODIS Data}

The Moderate Resolution Imaging Spectroradiometer (MODIS) has been widely used in large-scale eco-environment research because of its wide coverage area and short monitoring cycle. The normalized difference vegetation index (NDVI), land surface temperature (LST), and surface reflectivity are from MODIS remote sensing products from 2001 to 2018 (https: / / developers.google.com/earth-engine/datasets/catalog/modis, accessed on 2 February 2022). See Table 1 for specific product data.

Table 1. Research indicators and data products.

\begin{tabular}{ccccc}
\hline Index & Product & Spatial Resolution/m & Time Resolution/d & Indicator Description \\
\hline NDVI & MODIS13A2 & 1000 & 16 & The NDVI is based on BRDF correction data, and performs \\
various mask processes at the same time \\
$\begin{array}{c}\text { LST } \\
\text { Wetness }\end{array}$ & MODIS11A2 & 1000 & 8 & $\begin{array}{c}\text { LST is obtained by the most common split-window algorithm } \\
\text { MODIS09A1 provides bands 1 7 at a 500 m resolution in a } \\
\text { sinusoidal projected 8-day grid level 3 product }\end{array}$ \\
\hline
\end{tabular}


For the study area of this paper, we selected MODIS data from June to September of 2001, 2010, and 2018, and used the Google Earth Engine planetary cloud computing platform to pre-process different MODIS remote sensing images, such as atmospheric correction, stitching, cropping, and resampling. Meanwhile, we used the mean synthesis method to synthesize the MODIS data with different temporal resolutions to obtain the results available for the paper.

\subsubsection{Land-Use Data}

Land-use data (2001, 2010, and 2018) were obtained from the Resource and Environmental Science Data Center of the Chinese Academy of Sciences (http:/ / www.Resdc.cn, accessed on 2 February 2022). The spatial resolution was $1000 \mathrm{~m}$. The data from 2000 to 2010 were interpreted from Landsat TM/ETM, and the data from 2018 were interpreted from Landsat 8 (OLI/TIRS). Land use was divided into six categories: farmland, forest, grassland, water, urban, and other.

\subsubsection{Auxiliary Data}

The river and vector boundaries were obtained from the 1:250,000 basic database of the China National Geographic Information Resource Catalog Service System (https: / / www.webmap.cn, accessed on 2 February 2022).Digital elevation models (DEMs) were obtained from the Geospatial Data Cloud (http:/ / www.giscloud.cn, accessed on 2 February 2022). The spatial resolution was $90 \mathrm{~m}$.

\subsection{Methods}

\subsubsection{Construction of the CNLI}

(1) There is a huge difference in spatial resolution between the DMSP/OLS and the NPP/VIIRS nighttime light data. In order to effectively connect the DMSP/OLS and the NPP/VIIRS data, this paper refers to the relevant research results to desaturate the DMSP/OLS nighttime light data from 2001 to 2010 [35,36]. The calculation formula is as follows:

$$
V A N U I=1-N D V I \times N T L
$$

where NDVI is the normalized vegetation index; NTL is the normalized value of the DMSP/OLS night light data; and VANUI is the pixel value of the night light after desaturation.

(2) This paper uses a comprehensive night light index (CNLI) to reflect the level of urbanization and the intensity of social and economic activities [37]. To better show the development of towns within the administrative district, we create $3 \mathrm{~km} \times 3 \mathrm{~km}$ fishing grids and calculate the CNLI of each grid. The $C N L I$ is defined as the product of the light area proportion $(L A P)$ and the mean light intensity $(M L I)$ of a certain area. The calculation formula is as follows:

$$
\begin{gathered}
C N L I=L A P \times M L I \\
L A P=\frac{S_{\text {light }}}{S} \\
M L I=\frac{\sum_{i=1}^{63} N_{i} \times D N_{i}}{\sum_{i=1}^{63} N_{i} \times 63}
\end{gathered}
$$

where $S_{\text {light }}$ is the effective lighting area ratio; $S$ is the total area of the region; $D N_{i}$ is the light pixel value; and $N_{i}$ is the number of $D N_{i}$ pixels of light value.

\subsubsection{Construction of the RSEI}

This paper uses an RSEI to characterize the eco-environment in the Guanzhong region. The RSEI selects four ecological variables: greenness, heat, wetness, and dryness. Among them, greenness is represented by the NDVI; heat is represented by LST; wetness is 
represented by the wet component of the tasseled cap transformation; and the dryness is synthesized by the normalized difference build-up and soil index (NDBSI) [24].

$$
\begin{aligned}
& \text { Wetness }=0.1147 \text { Red }+0.2489 N I R 1+0.2408 \text { Blue }+0.3132 \text { Green }-0.3122 N I R 2 \\
& -0.6416 \text { SWIR } 1-0.5087 \text { WIR } 2 \\
& \qquad N D B S I=(B S I+I B I) / 2 \\
& B S I=\left[\left(S_{1}+\text { Red }\right)-(\text { Blue }+N I R)\right] /\left[\left(S_{1}+\text { Red }\right)+(\text { Blue }+N I R)\right] \\
& I B I=\left\{2 S_{1} /\left(S_{1}+N I R\right)-\left[N I R /(N I R+\text { Red })+\text { Green } /\left(\text { Green }+S_{1}\right)\right]\right\} / \\
& \left\{2 S_{1} /\left(S_{1}+N I R\right)+\left[N I R /(N I R+\text { Red })+\text { Green } /\left(\text { Green }+S_{1}\right)\right]\right\}
\end{aligned}
$$

where Red, NIR1, Blue, Green, NIR2, SWIR1, and SWIR2 are MODIS09A1 bands and DN represents the gray value of land surface temperature.

In order to make full use of the characteristics of the four ecological variables to construct the RSEI, this paper adopts principal component analysis (PCA) to realize the synthesis of the four variables. We used the normalized difference water index (NDWI) mask to remove the water area, avoiding the influence of a large water area on the RSEI. Finally, we used one to subtract the first principal component result to acquire the initial remote sensing ecological index value, $R S E I_{0}$. We use the "Min-max normalization" to normalize the initial remote sensing ecological index.

\subsubsection{Overall Coupling Trend Model}

The overall coupling trend model characterizes the coupling trend of the evolution of geographical elements by analyzing the distance and moving direction of the weighted centers of different geographical elements [38,39]. If the weighted center distance between two geographical elements is closer, the weighted trajectory of the weighted center between them is smaller, meaning that the overall coupling of the two geographical elements is higher. The calculation formula is as follows:

$$
\begin{gathered}
W C C=\left(\begin{array}{cc}
\frac{\sum_{i=1}^{n} \mathrm{~m}_{t i} X_{t i}}{\sum_{i=1}^{n} m_{t i}} & \frac{\sum_{i=1}^{n} m_{t i} Y_{t i}}{\sum_{i=1}^{n} m_{t i}}
\end{array}\right) \\
D=\sqrt{\left(X_{R S E I, t}-X_{C N L I, t}\right)^{2}+\left(Y_{R S E I, t}-Y_{C N L I, t}\right)^{2}} \\
\theta=\arccos \left(\frac{\Delta X_{R S E I} \times \Delta X_{C N L I}+\Delta Y_{R S E I} \times \Delta Y_{C N L I}}{\sqrt{\left(\Delta X_{R S E I}^{2}+\Delta Y_{C N L I}\right) \times\left(\Delta X_{C N L I}^{2}+\Delta Y_{R S E I}^{2}\right)}}\right) \times \pi
\end{gathered}
$$

where WCC is the weighted center coordinates of the RSEI or the CNLI in the Guanzhong region; $m_{t i}$ denotes the RSEI or CNLI value of pixel $i$ in period $t ; X_{t i}$ and $Y_{t i}$ are the central coordinates of pixel $i$ in period $t ; D$ is the spatial distance between the CNLI and RSEI weighted centers in period $t ; X_{R S E I, t}, Y_{R S E I, t}$ and $X_{C N L I, t}, Y_{C N L I, t}$ are the coordinates of the weighted centers of the CNLI and the NTL in period $t ; \theta$ is the angle between the moving trajectories of the RSEI and the CNLI weighted center in a certain period of time; and $\Delta X_{R S E I}, \Delta Y_{R S E I}$ and $\Delta X_{C N L I}, \Delta Y_{C N L I}$ are the change values of the RSEI and CNLI weighted center coordinates.

\subsubsection{Coupling Coordination Model}

The coupling and coordination of urban development and the eco-environment are important factors that affect the high-quality development of the Guanzhong region [40]. Therefore, this paper uses coupling coordination theory to construct a coupling coordination model of urban development and eco-environment, analyzes the trend of coupling 
coordination degree, and divides the types of coupling coordination. The coupling model is constructed as:

$$
\begin{gathered}
C=2 \times \sqrt{(N \times R) /(N+R)^{2}} \\
T=\alpha \times N+\beta \times R \\
D=\sqrt{C \times T}
\end{gathered}
$$

where $C$ is the coupling degree between urban development and the eco-environment; $N$ is the CNLI value; $R$ represents the RSEI; $T$ represents the comprehensive development evaluation index; $D$ is the coupling coordination degree, where the greater the value, the higher the coupling coordination level; and $\alpha$ and $\beta$ represent the weights of urban development and the eco-environment. This paper sets $\alpha=\beta=0.5$ because urban development is as important as the eco-environment.

Referring to the research results [41], the coupling coordination degree is divided into five levels by an equal interval segmentation method: senior coordination (0.8-1), slight coordination (0.6-0.8), reluctant coordination (0.4-0.6), slight disorder (0.2-0.4), and severe disorder (0-0.2). In addition, the coupling coordination types are classified according to the RSEI and the CNLI: eco-environment lag (N-R > 0.1), system coordinated development $(|\mathrm{N}-\mathrm{R}| \leq 0.1)$, and urban development lag $(\mathrm{N}-\mathrm{R}<-0.1)$.

\section{Results}

\subsection{Accuracy Verification \\ 3.1.1. NLI Inspection}

This work used ArcGIS to create a $3 \mathrm{~km} \times 3 \mathrm{~km}$ grid, and used grid cells to extract the average value of CNTL and RSEI pixels to realize grid data gridding in the Guanzhong region. This paper analyzes the correlation between the VANUI normalized values in 2001 and 2010 in addition to the NTL normalized values in 2018. In Figure 3, the abscissa is the VANUI normalized value in 2001 and 2010, the ordinate is the NTL normalized pixel value in 2018, and the points represent the number of grids. Figure 2 shows that the coefficient of determination, $R^{2}$, is 0.70 and 0.90 ; the correlations all passed the confidence test of 0.01 , indicating that the DMSP/OLS nighttime light values of desaturation in 2001 and 2010 and the NPP/VIIRS values in 2018 are temporally continuous.
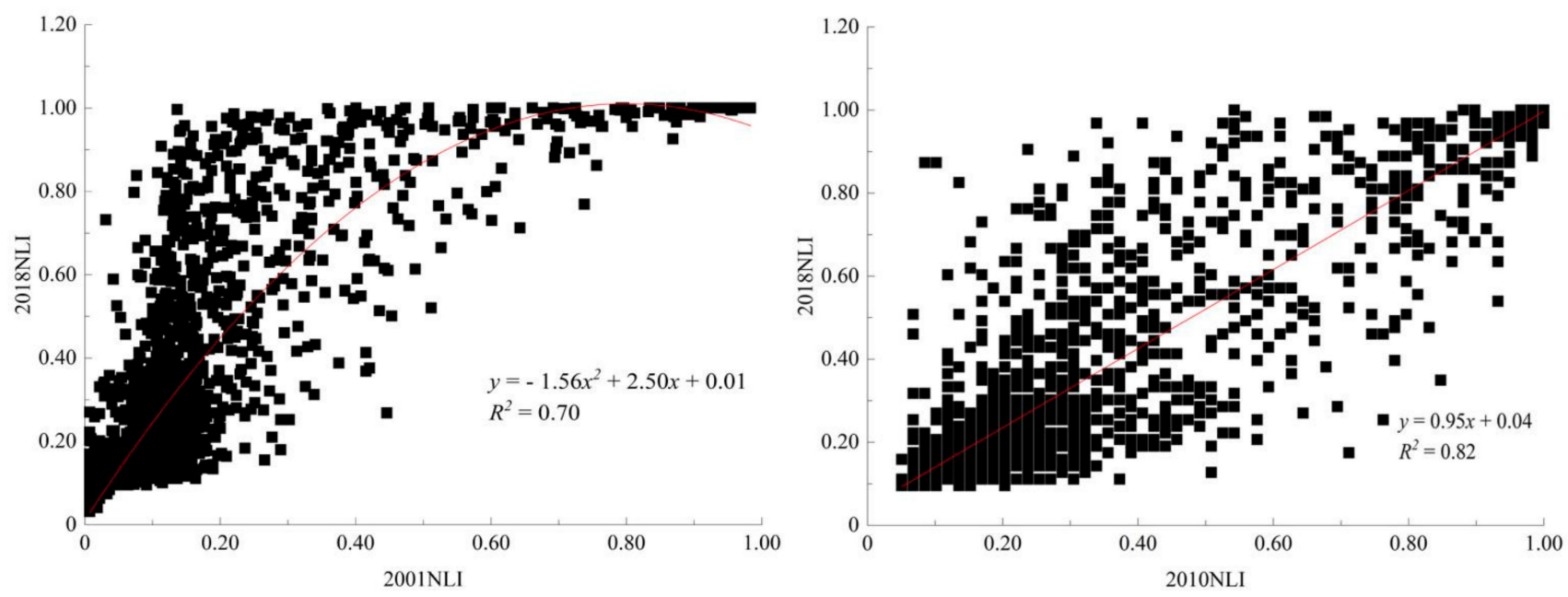

Figure 3. Night light desaturation treatment inspection.

\subsubsection{Principal Component Analysis Results}

Table 2 reveals that, during the research period, the contribution rate of the eigenvalues of the first principal component (PC1) is greater than $60 \%$, which indicates that the first principal component, compared with other principal components, concentrates most of the feature information of the four indicators. By analyzing the contribution rates of the 
four ecological variables to the RSEI, it is found that the contribution rates of the NDVI and wetness are positive, indicating that they have a positive impact on the regional eco-environment; the contribution rates of LST and dryness are negative, indicating that they have a negative effect on the quality of the eco-environment. Therefore, the RSEI constructed based on the first principal component can accurately reflect the overall status of the regional eco-environment.

Table 2. First principal component eigenvalues and contribution rates.

\begin{tabular}{cccc}
\hline Ecological Indicators & $\mathbf{2 0 0 1}$ & $\mathbf{2 0 1 0}$ & $\mathbf{2 0 1 8}$ \\
\hline NDVI & 0.52 & 0.53 & 0.53 \\
LST & -0.50 & -0.52 & -0.51 \\
Wetness & 0.43 & 0.35 & 0.45 \\
Dryness & -0.53 & -0.56 & -0.54 \\
Contribution rate & $79.45 \%$ & $63.48 \%$ & $75.61 \%$ \\
\hline
\end{tabular}

\subsection{Spatial-Temporal Distribution of Urban Development and the Eco-Environment}

\subsubsection{Guanzhong Region CNLI and Its Changes}

This study obtained CNLI images of the Guanzhong region to reflect the development status of regional towns. Figure 4 reveals that, from 2001 to 2018, the CNLI increased from 0.29 to 0.64 , indicating that the overall level of urban development in the Guanzhong region showed a rapid upward trend. The regional differences were obvious, gradually forming a belt-like distribution pattern with the main urban area of $\mathrm{Xi}^{\prime}$ an as the core. The scale expansion of urban development in the Guanzhong region was obvious, and the adjacent CNLI high-value areas continued to break the administrative boundaries and form larger high-value areas. However, the phenomenon that the high CNLI of the five prefecturelevel cities merge with each other was still not prominent. It shows that the connection between the administrative districts in the Guanzhong region has been continuously strengthened but that the development level of urban agglomeration integration needs to be further improved.
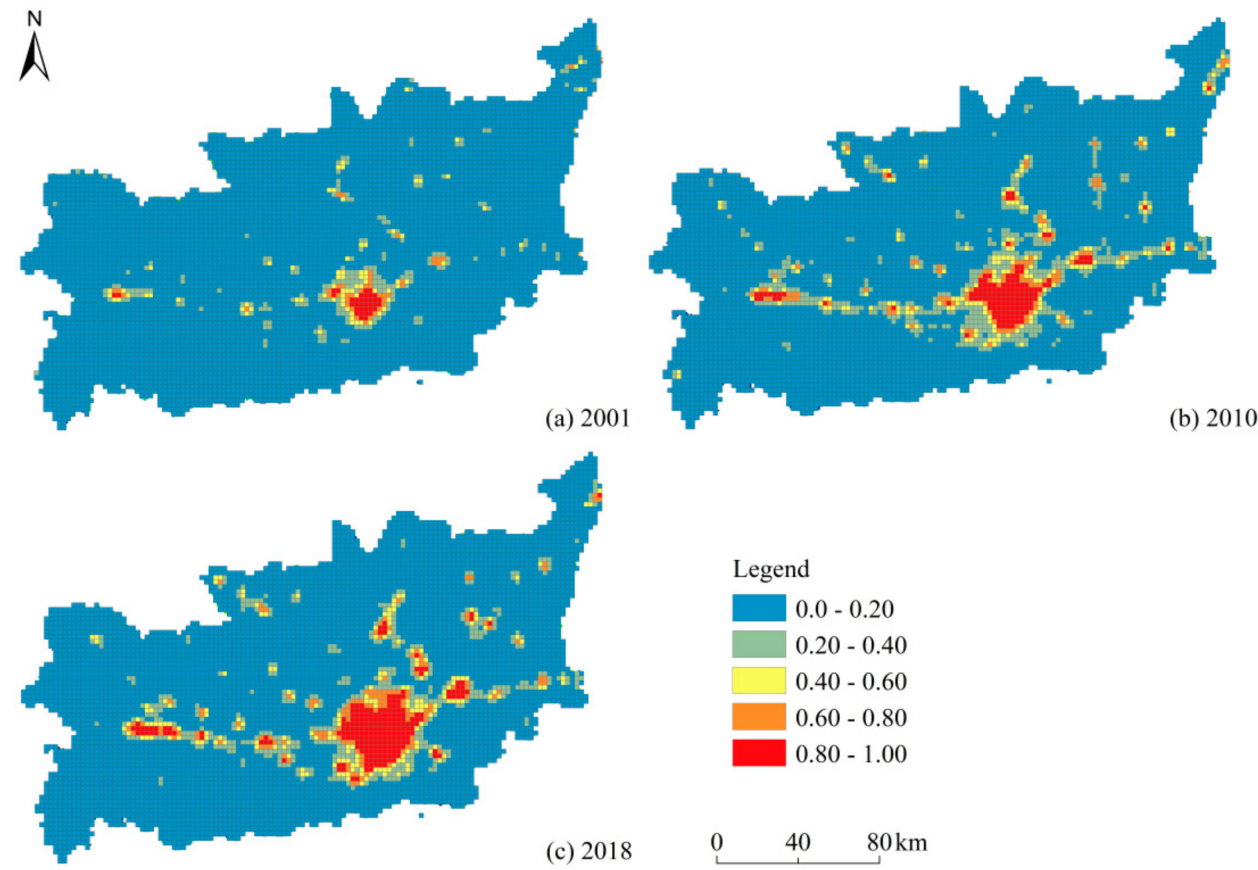

Figure 4. Spatial and temporal changes of the CNLI in the Guanzhong region from 2001 to 2018. (a) the CNLI in 2001; (b) the CNLI in 2010; (c) the CNLI in 2018. 
In order to improve the CNLI changes, we divided the CNLI into five levels: Very Low (0-0.2), Low (0.2-0.4), Medium (0.4-0.6), High (0.6-0.8), and Very High (0.8-1). The results (Table 3) show that the proportion of "Very Low" grids continued to decline (from 94.22\% to $84.62 \%$ ) and that the proportion of "Low", "Medium", "High", and "Very High" grids showed a rising trend (from $3.33 \%$ to $6.97 \%, 1.22 \%$ to $3.00,0.65 \%$ to $2.22 \%$, and $0.57 \%$ to $3.19 \%$, respectively). This demonstrates that the CNLI in the Guanzhong region has shown steady growth over the past 18 years, which indicates that the level of urban development has improved.

Table 3. The proportion of CNLI grades in the Guanzhong region from 2001 to 2018.

\begin{tabular}{cccc}
\hline Grade & $\mathbf{2 0 0 1}$ & $\mathbf{2 0 1 0}$ & $\mathbf{2 0 1 8}$ \\
\hline Very Low & $94.22 \%$ & $84.97 \%$ & $84.62 \%$ \\
Low & $3.33 \%$ & $8.05 \%$ & $6.97 \%$ \\
Medium & $1.22 \%$ & $2.83 \%$ & $3.00 \%$ \\
High & $0.65 \%$ & $1.78 \%$ & $2.22 \%$ \\
Very High & $0.57 \%$ & $2.38 \%$ & $3.19 \%$ \\
\hline
\end{tabular}

\subsubsection{Guanzhong Region RSEI and Its Changes}

Figure 5 shows the distribution of the RSEI in 2001, 2010, and 2018. The results of the RSEI in the Guanzhong region showed a slow upward trend from 2001 to 2018, but the overall eco-environment level was low. The RSEI value of the Guanzhong region in 2001 was the lowest, at only 0.52 , while the RSEI value of 2018 increased by about $13.46 \%$ to 0.59 , indicating that the eco-environment in the Guanzhong region has improved in the past 18 years. For the 2001 RSEI, the low values were mainly distributed in the central plains. In 2018, the low values of the RSEI decreased. Most of them were concentrated in built-up areas, such as the Beilin District and the Jintai District.
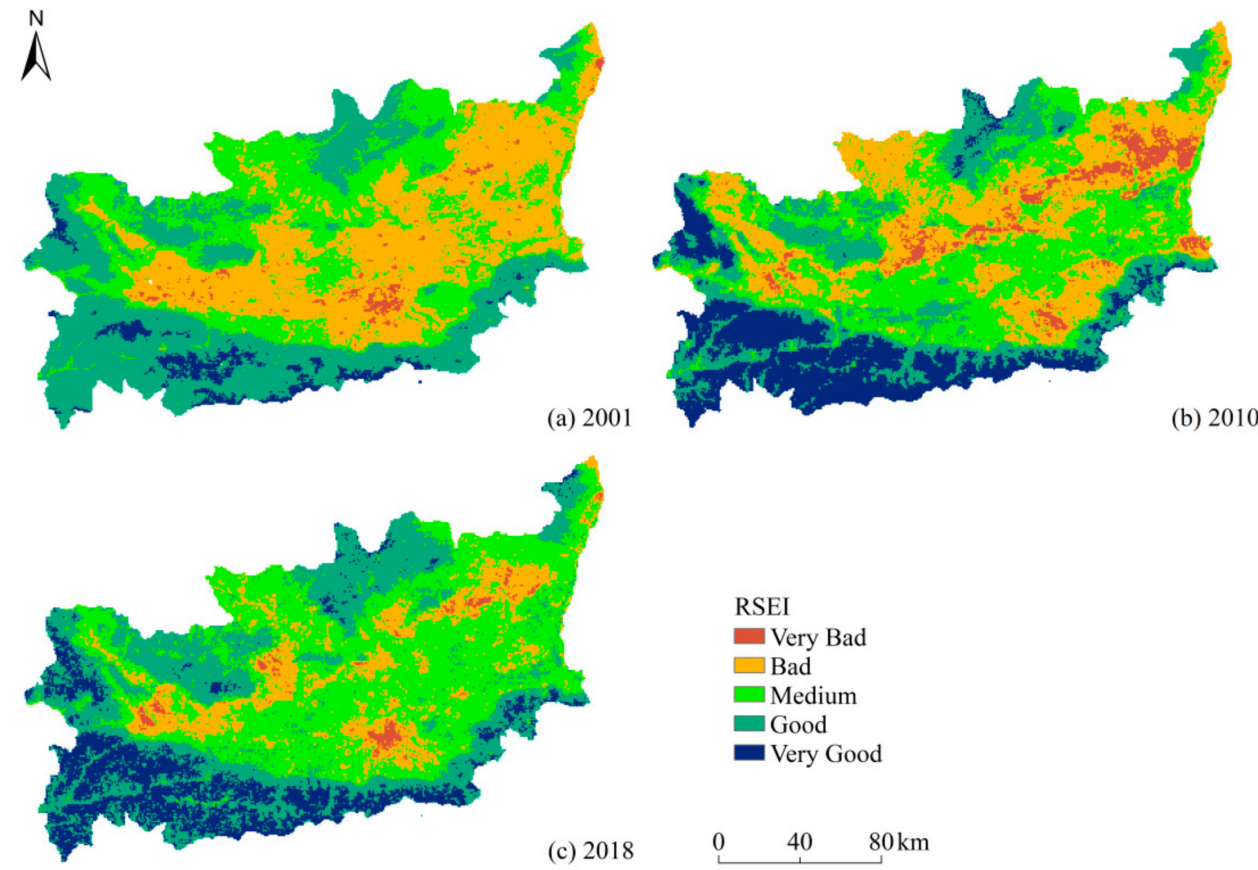

Figure 5. Spatial and temporal changes in the RSEI in the Guanzhong region from 2001 to 2018. (a) the RSEI in 2001; (b) the RSEI in 2010; (c) the RSEI in 2018.

In order to better reveal the spatial distribution characteristics of the RESI in the Guanzhong region this paper divides the RSEI into five classes according to an interval of 0.2 (Table 4). In the past 18 years, the total area in the Guanzhong region with ecological conditions classified as "Bad" and "Good" decreased (the proportion of the "Bad" grade 
decreased from $32.18 \%$ to $12.61 \%$, and the proportion of the "Good" grade decreased from $32.14 \%$ to $29.51 \%$ ). The total area of "Medium" and "Very Good" grades increased by $6141.25 \mathrm{~km}^{2}$ and $6095.52 \mathrm{~km}^{2}$, respectively. In 2001 the proportion of "Good" and "Bad" ecological conditions was close, accounting for $32 \%$ of the total area of the study area; "Medium" accounted for about 30\%. In 2018 the proportion of "Medium" areas was "42.01\%", which is vastly different compared with 2001. Changes in the proportion structure indicate that, in the past 18 years, about $13 \%$ of the areas in the "Good" grade rose to the "Very Good" grade, indicating that the ecological quality of the Guanzhong region has improved significantly.

Table 4. Area and proportion of RSEI grades in the Guanzhong region from 2001 to 2018.

\begin{tabular}{ccccccc}
\hline \multirow{2}{*}{ Grade } & \multicolumn{2}{c}{$\mathbf{2 0 0 1}$} & \multicolumn{2}{c}{$\mathbf{2 0 1 0}$} & \multicolumn{2}{c}{$\mathbf{2 0 1 8}$} \\
\cline { 2 - 7 } & Area/km & Percentage $\%$ & Area $/ \mathbf{k m}^{\mathbf{2}}$ & Percentage/\% & Area/km & Percentage/\% \\
\hline Very Bad & 645.87 & 1.17 & 2320.12 & 4.20 & 666.93 & 1.21 \\
Bad & $17,761.57$ & 32.18 & $14,196.09$ & 25.72 & 6960.49 & 12.61 \\
Medium & $17,044.6$ & 30.88 & $18,141.65$ & 32.87 & $23,185.85$ & 42.01 \\
Good & $17,741.96$ & 32.14 & $11,022.88$ & 19.97 & $16,285.21$ & 29.51 \\
Very Good & 2000.35 & 3.62 & 9513.61 & 17.24 & 8095.87 & 14.67 \\
\hline
\end{tabular}

\subsection{Spatial-Temporal Coupling between the CNLI and the RSEI}

\subsubsection{Temporal Coupling Trend of the CNLI and the RSEI}

This paper uses an overall coupling trend model to evaluate the coupling degree of the CNLI and the RSEI in the Guanzhong region. Overall, the weighted central distance and the moving direction angle of the CNLI and the RSEI in the Guanzhong region (Table 5) showed an overall decreasing trend during 2001-2018, indicating that the overall coupling between urban development and the eco-environment in the region was increasing. The main reason for this is that during the rapid development of regional urban, government departments focus on ecological protection and implement measures for ecological restoration. However, the weighted centers of the CNLI and the RSEI in the Guanzhong region are in opposition to each other. From 2001 to 2010 the weighted center of the CNLI shifted to the southeast and the weighted center of the RSEI shifted to the northeast. From 2010 to 2018 the weighted center of the CNLI shifted to the northeast and the weighted center of the RSEI shifted to the southwest. The reasons for these changes were mainly influenced by the policy of returning farmland to forest and grass, as well as the development of the Xi'an-Xianyang New Area.

Table 5. Overall coupling analysis of the CNLI and the RSEI in the Guanzhong region from 2001 to 2018.

\begin{tabular}{|c|c|c|c|c|c|c|c|}
\hline \multirow{3}{*}{ Year } & \multicolumn{4}{|c|}{ Weighted Center Coordinates } & \multirow{3}{*}{$\begin{array}{l}\text { Weighted Center } \\
\text { Distance/km }\end{array}$} & \multirow{3}{*}{ Period } & \multirow{3}{*}{ Angle Change ${ }^{\circ}$} \\
\hline & \multicolumn{2}{|c|}{ CNLI } & \multicolumn{2}{|c|}{ RSEI } & & & \\
\hline & Longitude $^{\circ}$ & Latitude $^{\circ}$ & Longitude $^{\circ}$ & Latitude $/^{\circ}$ & & & \\
\hline 2001 & 108.71 & 34.55 & 108.30 & 34.24 & 51.02 & 2001-2010 & 38.42 \\
\hline 2010 & 108.81 & 34.53 & 108.35 & 34.35 & 46.68 & 2010-2018 & 27.03 \\
\hline 2018 & 108.69 & 34.55 & 108.29 & 34.28 & 47.41 & 2001-2018 & 6.19 \\
\hline
\end{tabular}

\subsubsection{Spatial Coordination Characteristics of the CNLI and the RSEI}

In this paper a coordination coupling degree model is applied to calculate the coordination degree of the CNLI and the RSEI for three time periods: 2001, 2010, and 2018. The results (Figure 6) show that, from 2001 to 2018, the coordination degree between the CNLI and the RSEI in the Guanzhong region showed a continuous upward trend (from 0.22 to 0.41 ), the overall coupling coordination level was at a low level, and the type of coupling coordination gradually changed from slight imbalance to reluctantly coordinate, indicating that urban development and the eco-environment quality in the Guanzhong region tended to be in a coordinated development trend. Spatially, it shows a high central 
and low surrounding spatial distribution pattern. In terms of cities (Figure 7), the coordination degree of $\mathrm{Xi}^{\prime}$ an has always been at the forefront, followed by Xianyang and Weinan. Cities with a high degree of coordination and coupling pay attention to the improvement of the regional eco-environment while maintaining a rapid development of urbanization, achieving a virtuous cycle of urban development and the eco-environment. Cities with a low degree of coordination and coupling may be affected by their backward industrial structure, lower level of economic development, and low urbanization rate.
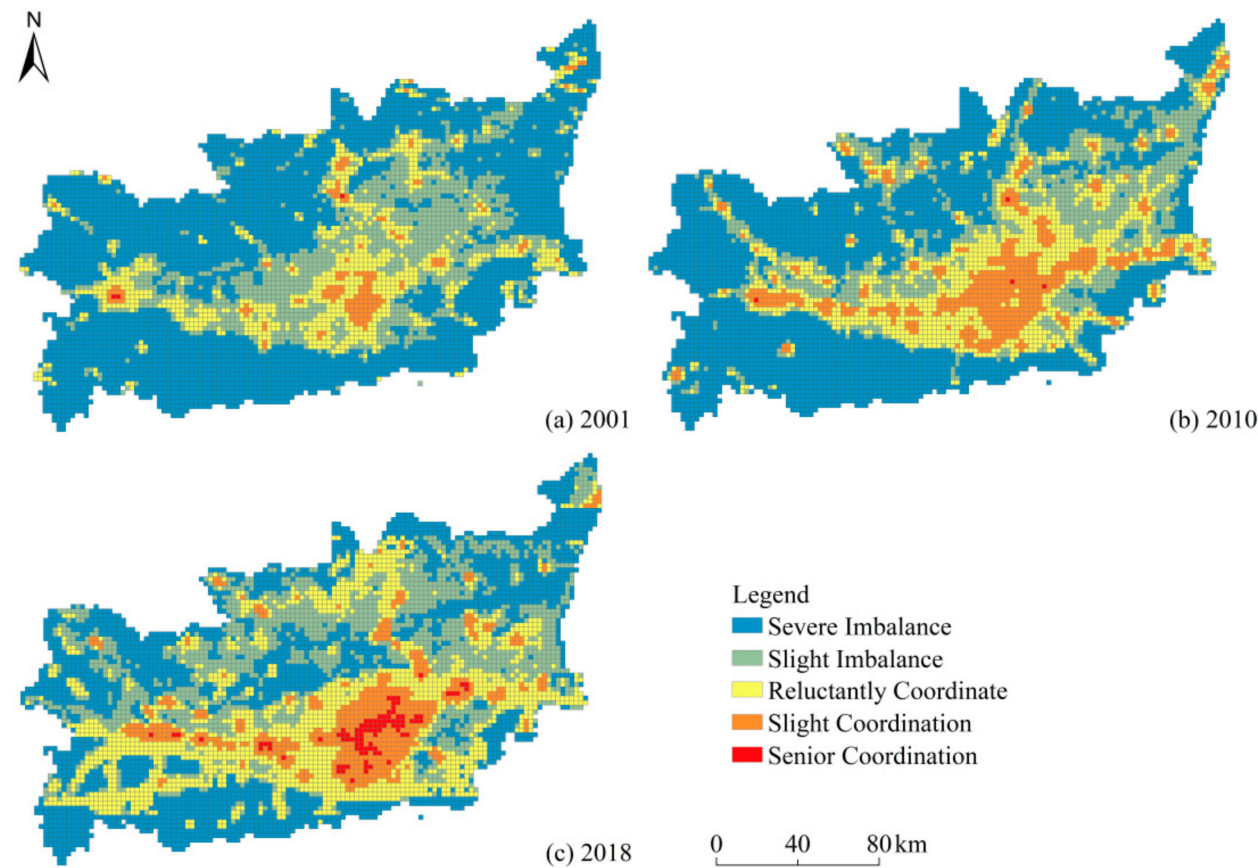

$$
\begin{aligned}
& \text { Legend } \\
& \text { Severe Imbalance } \\
& \text { Slight Imbalance } \\
& \text { Reluctantly Coordinate } \\
& \text { Slight Coordination } \\
& \text { Senior Coordination }
\end{aligned}
$$

(c) 2018

(b) 2010

Figure 6. Temporal and spatial changes in CNLI and RSEI coordination coupling in the Guanzhong region from 2001 to 2018. (a) the coordination coupling in 2001; (b) the coordination coupling in 2010; (c) the coordination coupling in 2018.

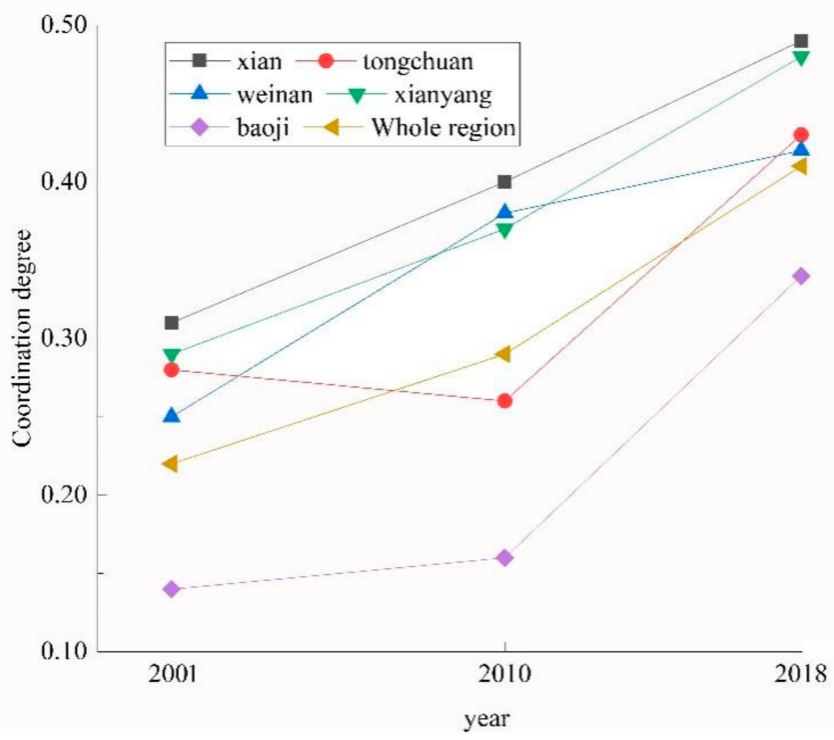

Figure 7. Changes in the level of coordination coupling in the five Guanzhong region cities.

From 2001 to 2018, Figure 8 shows that the sub-type of coordination coupling between the CNLI and the RSEI in the Guanzhong region gradually changed from balance of system to the coexistence of balance of system, ecological lag, and urban lag. With 
rapid socio-economic development and urban expansion, $\mathrm{Xi}^{\prime}$ an gradually shifted from balance of system to ecological lag. Before 2010, due to the over-exploitation of mineral resources, Tongchuan was mainly ecological lag. However, after 2010, mineral resources were increasingly depleted and Tongchuan began to adjust its industrial structure, focusing on the ecological restoration of mines, gradually transforming into balance of system. After 2001, with its rich tourism resources, Weinan gradually shifted from urban lag to balance of system. Xianyang always maintained balance of system, but the gap between urban development and the eco-environment is growing. Due to the large mountainous area in the region, which limits its urban development, Baoji has been in a state of urban lag. In conclusion, while the urban development process in the Guanzhong region accelerated from 2001 to 2018, the improvement of the eco-environment lagged relatively behind. Government departments need to focus on ecological civilization construction while promoting regional economic development.
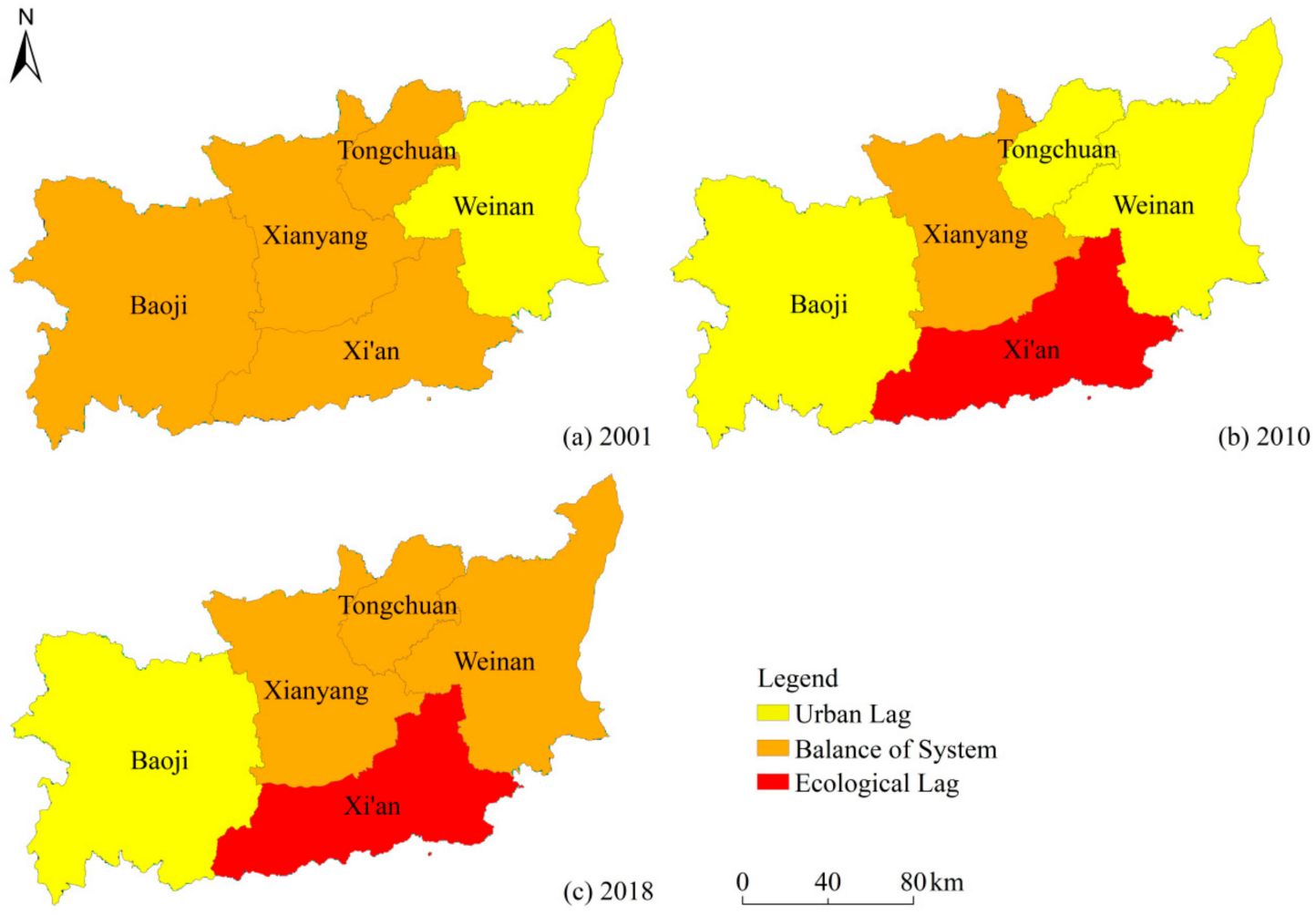

Figure 8. Time changes of sub-types of coordination coupling in the five Guanzhong region cities. (a) the sub-types of coordination coupling in 2001; (b) sub-types of coordination coupling in 2010; (c) the sub-types of coordination coupling in 2018.

\section{Discussion}

\subsection{Eco-Environment Changes in the Guanzhong Region}

As seen through the statistics of the RSEI average value of the Guanzhong region from 2001 to 2018, the overall eco-environment of the Guanzhong region is gradually improving. The average RSEI value has increased by $13.46 \%$, and the area with the eco-environment status of "Very Good" has increased by 304.72\%. However, it is an indisputable fact that the rapid development of urbanization and the replacement of the original natural surface by urban construction land will have a negative impact on the regional ecological environment [42]. However, the research results of this paper show that, from 2001 to 2018, with the rapid development of urbanization, the eco-environment of the Guanzhong region has improved somewhat, which is different from previous research results. In general, the relationship between urban development and ecological conditions is not simply negative, but is influenced by government policies and ecological restoration 
projects $[23,41]$. As human activities have a profound impact on regional ecosystem structures and spatial changes, the problem of global ecological environment deterioration has become increasingly serious and sustainable development has become one of the most important themes around the world $[43,44]$. As the world's largest developing country and the world's second largest economy, China has formulated a series of ecological protection measures and economic development guidelines since the beginning of the 21st century, such as the policy of returning farmland to grassland, in order to achieve regional highquality development $[23,45]$. An interesting example comes from the Guanzhong region, from 2001 to 2018, the area of returning farmland to forests and grasslands was $985.77 \mathrm{~km}^{2}$, accounting for $1.78 \%$ of the area of the Guanzhong region. To better analyze the impact of the policy of returning farmland to forests and grasses on the regional eco-environment, we compared the RSEI of the area returned to forests and grasses with that of the whole region and found that the mean RSEI value of the area returned to forests and grasses (0.62) was higher than the mean value of the whole region (0.59), and the RSEI variability of the area returned to forests and grasses $(0.56 \%)$ was significantly higher than that of the whole region $(0.44 \%)$. Therefore, we can speculate that, since 2001, the Guanzhong region has improved regional ecological environment conditions through human intervention and regulation, forming an upward trend of the RSEI in the study area.

In addition, the low RSEI value area in the Guanzhong region gradually shifted to urban areas. Table 6 shows that the eco-environmental quality of urban construction land shows a decreasing trend from 2001 to 2018, which was due to the exposed surface, many man-made buildings, and sparse vegetation. In contrast, with the reduction in the RSEI in urban areas, the quality of the ecological environment of a large amount of cultivated land and bare land has been improved. This phenomenon is especially obvious in the main urban areas of $\mathrm{Xi}^{\prime}$ an.

Table 6. RSEI values of different land-use types.

\begin{tabular}{cccc}
\hline Class & $\mathbf{2 0 0 1}$ & $\mathbf{2 0 1 0}$ & $\mathbf{2 0 1 8}$ \\
\hline Farmland & 0.41 & 0.42 & 0.49 \\
Forest & 0.69 & 0.76 & 0.76 \\
Grassland & 0.58 & 0.59 & 0.65 \\
Urban & 0.32 & 0.30 & 0.31 \\
Other & 0.40 & 0.47 & 0.48 \\
\hline
\end{tabular}

\subsection{Urbanization Process of the Guanzhong Region}

Since 2000, China has put forward the Western Development Plan and has increased its support for the Guanzhong region. The establishment of various economic zones and high-tech zones in the region has accelerated the process of urban development. The rapid growth of the CNLI is a reflection of the rapid urban development and economic development in the Guanzhong region over the past 18 years. However, affected by the long-standing tradition of unipolar urban development in $\mathrm{Xi}^{\prime}$ an, the differences in the level of urban development in the Guanzhong region are still prominent. The gap between second-tier cities, such as Baoji, Xianyang, Weinan, and Tongchuan, and the primary city of Xi'an in urban development still exists. However, Shaanxi Province has promoted the integration of Xi'an and Xianyang and focused on the development of Baoji, Weinan, and Tongchuan in recent years. The Guanzhong region has gradually formed a "multi-city growth pole" situation, narrowing the differences in regional urbanization and driving the overall coordinated development of the region.

\subsection{Interaction between Urban Development and the Eco-Environment}

The relationship between urban development and the eco-environment is quite complicated. Urbanization limits the improvement of the quality of the regional eco-environment, and the eco-environment also has certain constraints on urban development [46]. In particular, with the rapid economic development in China since the 21st century, the level of 
urbanization in the central and western regions has also gradually increased. It is worth noting that the research results of this paper show that the degree of coordination and coupling between urban development and the eco-environment in the Guanzhong region is continuously improving. At present, high-quality regional development has become mainstream. As mentioned above, the Chinese government has continuously formulated various policies to improve the regional eco-environment while the social economy develops rapidly. Although the eco-environment in the Guanzhong region shows a trend of improvement, our research finds that this improvement mainly comes from non-urbanized areas rather than urban construction land areas. The reduction in the RSEI in the builtup area shows the limitations of current eco-environmental protection measures in the Guanzhong region [23].

At present, China is in a stage of rapid urbanization, and the large number of people flowing into cities has led to an increasing demand for urban construction land, making the scale of urban construction land in China expand rapidly [47]. With the further deepening of the Western Development Plan a large number of domestic resources are tilted to the west, and the future expansion of urban construction land is still an important trend for urban development in the western region. The demand for land in the process of urban development is still severe, resulting in constraints of urban development on the ecoenvironment existing for long periods of time. Therefore, how to optimize the allocation of land resources and coordinate the relationship between urban development and the eco-environment is an important task in national land space planning. In order to achieve regional high-quality development, it is necessary to pay more attention to improving the internal eco-environment and optimizing urban ecological land while expanding the scale of urban construction land.

\subsection{Limitations and Prospects}

The RSEI proposed by $\mathrm{Xu}$ et al. [24] analyzes the regional eco-environment quality with raster image elements as the research object, which can better reflect the spatial variation in the eco-environment quality within administrative regions. Most of the urban land in the Guanzhong region is concentrated in the Weihe River basin, but this paper uses the normalized difference water index (NDWI) to remove waters in order to avoid the influence of water on the RSEI. Therefore, it is suggested to increase the evaluation system of water eco-environments in future research, processes which comprehensively evaluate the ecological environment quality of the Guanzhong region should be employed.

Although the RSEI and the CNLI have been widely used in eco-environment monitoring and urbanization development research, the interrelationship between urban development and eco-environment status still cannot be reflected due to the limitations of remote sensing data in terms of spatial and temporal resolution. Some scholars have combined macro and micro to propose a new development theory of nature and city [48]. Therefore, we can measure the relationship between urban development and the eco-environment at different levels (mega-cities, big cities, etc.) and from different perspectives (macro, micro, etc.) in subsequent studies.

\section{Conclusions}

This paper uses night light data and MODIS remote sensing data to evaluate urban development and the eco-environment in the Guanzhong region, as well as to analyze the coupling relationship between them. The results of this study show that: (1) From 2001 to 2018, the CNLI in the Guanzhong region has increased steadily, the level of urban development has gradually improved, and the connection between intra-regional cities has continuously strengthened. (2) The RSEI in the Guanzhong region gradually increased during the study period and the eco-environmental conditions have improved, but the overall level was low. (3) Although the overall coupling situation and degree of coordination between urban development and the eco-environment in the Guanzhong region were increasing, the eco-environment lagged behind urban development. While promoting 
regional economic development, it is necessary to pay attention to the construction of ecological civilization.

Author Contributions: Writing—original draft, J.W. and M.Z.; writing—review and editing, M.Z.; formal analysis and resources, W.Z.; methodology, C.Z.; data curation, C.Z. and J.W.; software, J.L. All authors have read and agreed to the published version of the manuscript.

Funding: This research was funded by the National Natural Science Foundation of China, grant number 41271284 .

Institutional Review Board Statement: Not applicable.

Informed Consent Statement: Not applicable.

Data Availability Statement: The data presented in this study are available within the article.

Conflicts of Interest: The authors declare no conflict of interest.

\section{References}

1. Wu, Z.; Zhang, Q.; Song, C.; Zhang, F.; Zhu, X.; Sun, P.; Fan, K.; Yu, H.; Shen, Z. Impacts of urbanization on spatio-temporal variations of temperature over the Pearl River Delta. Acta Geograph. Sin. 2019, 74, 2342-2357.

2. Ma, Y.; Zhao, M.; Li, J.; Wang, J.; Hu, L. Cooling Effect of Different Land Cover Types: A Case Study in Xi'an and Xianyang, China. Sustainability 2021, 13, 1099. [CrossRef]

3. Deng, X.; Huang, J.; Rozelle, S.; Zhang, J.; Li, Z. Impact of urbanization on cultivated land changes in China. Land Use Policy 2015, 45, 1-7. [CrossRef]

4. Song, X.; Hansen, M.C.; Stehman, S.V.; Potapov, P.V.; Tyukavina, A.; Vermote, E.F.; Townshend, J.R. Global land change from 1982 to 2016. Nature 2018, 560, 639-643. [CrossRef]

5. Xu, D.; Yang, F.; Yu, L.; Zhou, Y.; Li, H.; Ma, J.; Huang, J.; Wei, J.; Xu, Y.; Zhang, C.; et al. Quantization of the coupling mechanism between eco-environmental quality and urbanization from multisource remote sensing data. J. Clean. Prod. 2021, 321, 128948. [CrossRef]

6. Fan, W.; Wang, H.; Liu, Y.; Liu, H. Spatio-temporal variation of the coupling relationship between urbanization and air quality: A case study of Shandong Province. J. Clean. Prod. 2020, 272, 122812. [CrossRef]

7. Fang, C.; Liu, H.; Wang, S. The coupling curve between urbanization and the eco-environment: China's urban agglomeration as a case study. Ecol. Indic. 2021, 130, 108107. [CrossRef]

8. He, L.; Zhang, X.; Yan, Y. Heterogeneity of the Environmental Kuznets Curve across Chinese cities: How to dance with 'shackles'? Ecol. Indic. 2021, 130, 108128. [CrossRef]

9. Tang, P.; Huang, J.; Zhou, H.; Fang, C.; Zhan, Y.; Huang, W. Local and telecoupling coordination degree model of urbanization and the eco-environment based on RS and GIS: A case study in the Wuhan urban agglomeration. Sustain. Cities Soc. 2021, 75, 103405. [CrossRef]

10. Ahmad, M.; Jiang, P.; Murshed, M.; Shehzad, K.; Akram, R.; Cui, L.; Khan, Z. Modelling the dynamic linkages between ecoinnovation, urbanization, economic growth and ecological footprints for G7 countries: Does financial globalization matter? Sustain. Cities Soc. 2021, 70, 102881. [CrossRef]

11. Dong, L.; Longwu, L.; Zhenbo, W.; Liangkan, C.; Faming, Z. Exploration of coupling effects in the Economy-Society-Environment system in urban areas: Case study of the Yangtze River Delta Urban Agglomeration. Ecol. Indic. 2021, 128, 107858. [CrossRef]

12. Li, W.; Wang, Y.; Xie, S.; Cheng, X. Coupling coordination analysis and spatiotemporal heterogeneity between urbanization and ecosystem health in Chongqing municipality, China. Sci. Total Environ. 2021, 791, 148311. [CrossRef]

13. Geng, Y.; Zhang, H. Coordination assessment of environment and urbanization: Hunan case. Environ. Monit. Assess 2020, $192,637$. [CrossRef]

14. Liu, N.; Liu, C.; Xia, Y.; Da, B. Examining the coordination between urbanization and eco-environment using coupling and spatial analyses: A case study in China. Ecol. Indic. 2018, 93, 1163-1175. [CrossRef]

15. Cui, D.; Chen, X.; Xue, Y.; Li, R.; Zeng, W. An integrated approach to investigate the relationship of coupling coordination between social economy and water environment on urban scale-A case study of Kunming. J. Environ. Manag. 2019, 234, 189-199. [CrossRef]

16. Fang, C.; Cui, X.; Li, G.; Bao, C.; Wang, Z.; Ma, H.; Sun, S.; Liu, H.; Luo, K.; Ren, Y. Modeling regional sustainable development scenarios using the Urbanization and Eco-environment Coupler: Case study of Beijing-Tianjin-Hebei urban agglomeration, China. Sci. Total Environ. 2019, 689, 820-830. [CrossRef]

17. Fu, S.; Zhuo, H.; Song, H.; Wang, J.; Ren, L. Examination of a coupling coordination relationship between urbanization and the eco-environment: A case study in Qingdao, China. Environ. Sci. Pollut. Res. 2020, 27, 23981-23993. [CrossRef]

18. Lu, Y.; Kong, L.; Yan, L. Measurement on the coordination degree of China's urbanization and ecological environment. Urban Issues 2019, 13-22. 
19. Cai, J.; Li, X.; Liu, L.; Chen, Y.; Wang, X.; Lu, S. Coupling and coordinated development of new urbanization and agro-ecological environment in China. Sci. Total Environ. 2021, 776, 145837. [CrossRef]

20. Ren, F.; Yu, X. Coupling analysis of urbanization and ecological total factor energy efficiency-A case study from Hebei province in China. Sustain. Cities Soc. 2021, 74, 103183. [CrossRef]

21. Shao, Z.; Ding, L.; Li, D.; Altan, O.; Huq, M.E.; Li, C. Exploring the Relationship between Urbanization and Ecological Environment Using Remote Sensing Images and Statistical Data: A Case Study in the Yangtze River Delta, China. Sustainability 2020, 12, 5620. [CrossRef]

22. Feng, Y.; He, S.; Li, G. Interaction between urbanization and the eco-environment in the Pan-Third Pole region. Sci. Total Environ. 2021, 789, 148011. [CrossRef]

23. Zheng, Z.; Wu, Z.; Chen, Y.; Yang, Z.; Marinello, F. Exploration of eco-environment and urbanization changes in coastal zones: A case study in China over the past 20 years. Ecol. Indic. 2020, 119, 106847. [CrossRef]

24. $\mathrm{Hu}, \mathrm{X}$; $\mathrm{Xu}, \mathrm{H}$. A new remote sensing index for assessing the spatial heterogeneity in urban ecological quality: A case from Fuzhou City, China. Ecol. Indic. 2018, 89, 11-21. [CrossRef]

25. Zhao, W.; Yan, T.; Ding, X.; Peng, S.; Chen, H.; Fu, Y.; Zhou, Z. Response of ecological quality to the evolution of land use structure in Taiyuan during 2003 to 2018. Alex. Eng. J. 2021, 60, 1777-1785. [CrossRef]

26. Yang, X.; Bai, Y.; Che, L.; Qiao, F.; Xie, L. Incorporating ecological constraints into urban growth boundaries: A case study of ecologically fragile areas in the Upper Yellow River. Ecol. Indic. 2021, 124, 107436. [CrossRef]

27. Liu, C.; Yang, M.; Hou, Y.; Zhao, Y.; Xue, X. Spatiotemporal evolution of island ecological quality under different urban densities: A comparative analysis of Xiamen and Kinmen Islands, southeast China. Ecol. Indic. 2021, 124, 107438. [CrossRef]

28. Chen, Y.; Zheng, Z.; Wu, Z.; Qian, Q. Review and prospect of application of nighttime light remote sensing data. Prog. Geogr. 2019, 38, 205-223.

29. $\mathrm{Du}, \mathrm{Z}$; $\mathrm{Li}, \mathrm{X}$. Growth or shrinkage: New phenomena of regional development in the rapidly-urbanising Pearl River Delta. Acta Geogr. Sin. 2017, 72, 1800-1811.

30. Pan, W.; Fu, H.; Zheng, P. Regional Poverty and Inequality in the Xiamen-Zhangzhou-Quanzhou City Cluster in China Based on NPP/VIIRS Night-Time Light Imagery. Sustainability 2020, 12, 2547. [CrossRef]

31. Tan, M.; Li, X.; Li, S.; Xin, L.; Wang, X.; Li, Q.; Li, W.; Li, Y.; Xiang, W. Modeling population density based on nighttime light images and land use data in China. Appl. Geogr. 2018, 90, 239-247. [CrossRef]

32. Liu, H.; Ma, L.; Xu, L. Estimating spatiotemporal dynamics of county-level fossil fuel consumption based on integrated nighttime light data. J. Clean. Prod. 2021, 278, 123427. [CrossRef]

33. Zheng, Y.; He, Y.; Zhou, Q.; Wang, H. Quantitative Evaluation of Urban Expansion using NPP-VIIRS Nighttime Light and Landsat Spectral Data. Sustain. Cities Soc. 2022, 76, 103338. [CrossRef]

34. Chen, Y.; Li, Z.; Li, P.; Zhang, Z.; Zhang, Y. Identification of Coupling and Influencing Factors between Urbanization and Ecosystem Services in Guanzhong, China. Sustainability 2021, 13, 10637. [CrossRef]

35. Lin, Z.; Xu, H.; Chen, H. Urban heat island change and its relationship to the urbanization of three major urban agglomerations in China's eastern coastal region. Res. Environ. Sci. 2018, 31, 1695-1704.

36. Chen, S.; Hu, D.; Wong, M.S.; Ren, H.; Cao, S.; Yu, C.; Ho, H.C. Characterizing spatiotemporal dynamics of anthropogenic heat fluxes: A 20-year case study in Beijing-Tianjin-Hebei region in China. Environ. Pollut. 2019, 249, 923-931. [CrossRef]

37. Chen, J.; Zhuo, L.; Shi, P.J.; Toshiaki, I. The study on urbanization process in China based on DMSP/OLS data: Development of a light index for urbanization level estimation. J. Remote Sens. 2003, 7, 168-175.

38. Yang, C.; Zhan, Q.; Gao, S.; Liu, H. How Do the Multi-Temporal Centroid Trajectories of Urban Heat Island Correspond to Impervious Surface Changes: A Case Study in Wuhan, China. Int. J. Environ. Res. Public Health 2019, 16, 3865. [CrossRef]

39. Yang, Z.; Chen, Y.; Qian, Q.; Wu, Z.; Zheng, Z.; Huang, Q. The coupling relationship between construction land expansion and high-temperature area expansion in China's three major urban agglomerations. Int. J. Remote Sens. 2019, 40, 6680-6699. [CrossRef]

40. Shen, L.; Huang, Y.; Huang, Z.; Lou, Y.; Ye, G.; Wong, S. Improved coupling analysis on the coordination between socio-economy and carbon emission. Ecol. Indic. 2018, 94, 357-366. [CrossRef]

41. Ariken, M.; Zhang, F.; Liu, K.; Fang, C.; Kung, H. Coupling coordination analysis of urbanization and eco-environment in Yanqi Basin based on multi-source remote sensing data. Ecol. Indic. 2020, 114, 106331. [CrossRef]

42. Du, X.; Huang, Z. Ecological and environmental effects of land use change in rapid urbanization: The case of hangzhou, China. Ecol. Indic. 2017, 81, 243-251. [CrossRef]

43. Ramel, C.; Rey, P.; Fernandes, R.; Vincent, C.; Cardoso, A.R.; Broennimann, O.; Pellissier, L.; Pradervand, J.; Ursenbacher, S.; Schmidt, B.R.; et al. Integrating ecosystem services within spatial biodiversity conservation prioritization in the Alps. Ecosyst. Serv. 2020, 45, 101186. [CrossRef]

44. Borie, M.; Gustafsson, K.M.; Obermeister, N.; Turnhout, E.; Bridgewater, P. Institutionalising reflexivity? Transformative learning and the Intergovernmental science-policy Platform on Biodiversity and Ecosystem Services (IPBES). Environ. Sci. Policy 2020, 110, 71-76. [CrossRef]

45. Sun, Y.; Li, H. Data mining for evaluating the ecological compensation, static and dynamic benefits of returning farmland to forest. Environ. Res. 2021, 201, 111524. [CrossRef]

46. Sun, C.; Zhang, S.; Song, C.; Xu, J.; Fan, F. Investigation of Dynamic Coupling Coordination between Urbanization and the Eco-Environment-A Case Study in the Pearl River Delta Area. Land 2021, 10, 190. [CrossRef] 
47. Zheng, H.; Khan, Y.A.; Abbas, S.Z. Exploration on the coordinated development of urbanization and the eco-environmental system in central China. Environ. Res. 2022, 204, 112097. [CrossRef]

48. D'Acci, L. A new type of cities for liveable futures. Isobenefit Urbanism morphogenesis. J. Environ. Manag. 2019, 246, 128-140. [CrossRef] 